ARTICLE

https://doi.org/10.1038/s41467-019-09705-w

\title{
Formation of colloidal alloy semiconductor CdTeSe magic-size clusters at room temperature
}

\author{
Dong Gao', Xiaoyu Hao', Nelson Rowell ${ }^{2}$, Theo Kreouzis (10 3,4, David J. Lockwood², Shuo Han ${ }^{1}$, Hongsong Fan ${ }^{5}$,
} Hai Zhang ${ }^{5}$, Chunchun Zhang ${ }^{6}$, Yingnan Jiang ${ }^{7}$, Jianrong Zeng ${ }^{8,9}$, Meng Zhang (1) ${ }^{1} \&$ Kui Yu $u^{1,5,10}$

\begin{abstract}
Alloy semiconductor magic-size clusters (MSCs) have received scant attention and little is known about their formation pathway. Here, we report the synthesis of alloy CdTeSe MSC399 (exhibiting sharp absorption peaking at $399 \mathrm{~nm}$ ) at room temperature, together with an explanation of its formation pathway. The evolution of MSC-399 at room temperature is detected when two prenucleation-stage samples of binary CdTe and CdSe are mixed, which are transparent in optical absorption. For a reaction consisting of $\mathrm{Cd}$, Te, and Se precursors, no MSC-399 is observed. Synchrotron-based in-situ small angle X-ray scattering (SAXS) suggests that the sizes of the two samples and their mixture are similar. We argue that substitution reactions take place after the two binary samples are mixed, which result in the formation of MSC-399 from its precursor compound (PC-399). The present study provides a room-temperature avenue to engineering alloy MSCs and an in-depth understanding of their probable formation pathway.
\end{abstract}

\footnotetext{
${ }^{1}$ Institute of Atomic and Molecular Physics, Sichuan University, 610065 Chengdu, P. R. China. ${ }^{2}$ Metrology Research Centre, National Research Council of Canada, Ottawa, ON K1A OR6, Canada. ${ }^{3}$ School of Physics and Astronomy, Queen Mary University of London, London E1 4NS, UK. ${ }^{4}$ School of Physical Science and Technology, Sichuan University, 610065 Chengdu, P. R. China. ${ }^{5}$ Engineering Research Center in Biomaterials, Sichuan University, 610065 Chengdu, P. R. China. ${ }^{6}$ Analytical \& Testing Center, Sichuan University, 610065 Chengdu, P. R. China. ${ }^{7}$ Jilin Ginseng Academy, Changchun University of Chinese Medicine, 130117 Changchun, P. R. China. ${ }^{8}$ Shanghai Synchrotron Radiation Facility, Shanghai Advanced Research Institute, Chinese Academy of Sciences, 201204 Shanghai, P. R. China. ${ }^{9}$ Shanghai Institute of Applied Physics, Chinese Academy of Sciences, 201800 Shanghai, P. R. China. ${ }^{10}$ State Key Laboratory of Polymer Materials Engineering, Sichuan University, 610065 Chengdu, P. R. China. These authors contributed equally: Dong Gao, Xiaoyu Hao. Correspondence and requests for materials should be addressed to J.Z. (email: zengjianrong@sinap.ac.cn) or to M.Z. (email: mengzhang@scu.edu.cn) or to K.Y. (email: kuiyu@scu.edu.cn)
} 
C olloidal alloy semiconductor magic-size clusters (MSCs) have been reported only in a very limited fashion ${ }^{1,2}$, and their syntheses have been acknowledged to be quite challenging via conventional hot-injection and heating-up approaches $^{3-5}$. For the synthesis of metal (M) chalcogenide (E) semiconductor alloys, such as $\mathrm{ZnCdSe}^{1}$ and $\mathrm{CdTeSe}^{2}$ MSCs and CdTeSe quantum dots (QDs) ${ }^{6,7}$, corresponding $M$ and $E$ precursors are usually placed together in a reaction flask. The optical absorption reported of the $\mathrm{ZnCdSe}$ and CdTeSe MSCs seems to consist of two electronic transitions in the range of 300 to $350 \mathrm{~nm}$ for the former ${ }^{1}$, and at 464 and $520 \mathrm{~nm}$ for the latter ${ }^{2}$.

With relatively uniform size distributions, MSCs exhibit relatively narrow optical absorption, compared to corresponding $\mathrm{QDs}^{8-15}$. Very recently, a two pathway model has been proposed to be available in the prenucleation stage ${ }^{16}$, which is also called the induction period (occurring prior to nucleation and growth of QDs $)^{17}$. It is suggested that one pathway follows the LaMer model of the classical nucleation theory $(\mathrm{CNT})^{18-20}$, which involves monomers and fragments for which M-E covalent bonds form. The other pathway is argued to start with the self-assembly of $M$ and $\mathrm{E}$ precursors followed by the formation of $\mathrm{M}-\mathrm{E}$ covalent bonds inside the assembled species, each of which results in a special precursor compound (PC) of $\mathrm{MSCs}^{21}$. The PC (in a conventional solvent such as toluene or hexane or cyclohexane) is transparent in optical absorption, and has the character that one PC molecule can transform into a corresponding MSC (following first-order reaction kinetics $)^{22,23}$. The two pathways are linked by a MSC $\rightarrow$ PC transformation and PC fragmentation to QDs ${ }^{16}$. Usually, PCs form before monomers and fragments do (when the concentrations of $\mathrm{M}$ and $\mathrm{E}$ precursors in a reaction are not too low $)^{21}$.

The two pathway model indicates the distinct possibility of a selective two-step approach to the exclusive production of MSCs in a single ensemble form, at the same time without the complication of the presence of QDs ${ }^{24}$. The first step is to produce the PC at a relatively high temperature but still within the induction period. The second step is to form MSCs at a lower temperature, such as room temperature, from the PCs produced in an induction period sample. This two-step method has some definite and obvious differences to the conventional hot-injection and heating-up approaches ${ }^{3-5}$, and has been validated to be efficient in producing binary CdTe MSC- $371^{23,24}$, CdSe MSC- $415^{25}$, ZnSe MSC-29921, CdS MSC-311 16,22,26, and MSC-322 26 . These MSCs have distinctive sharp optical absorption peaks at the wavelength of $\sim 371,415,299,311$, and $322 \mathrm{~nm}$, respectively. However, there has been no report on alloy ternary MSCs via the selective twostep approach, and the formation pathway of alloy MSCs remains largely unexplored.
Chalcogenide (E) anion exchange reactions have been reported for semiconductor nanoparticles $(\mathrm{NPs})^{27-31}$, so have cation exchange reactions ${ }^{32-36}$. Such chemical transformations have been accepted to be promising alternatives to producing colloidal NPs with improved control over composition and morphology. For example, E-based partial anion exchange reactions have been reported, which allow the preparation of $\mathrm{CdS} / \mathrm{CdTe}$ heterodimers from CdS (with tri- $n$-octylphosphine telluride $\left(\mathrm{Te}=\mathrm{P}\left(\mathrm{C}_{8} \mathrm{H}_{17}\right)_{3}\right.$, TeTOP at $\left.260{ }^{\circ} \mathrm{C}\right)^{27}$, and ternary CdTeS NPs from CdTe (with $\mathrm{Na}_{2} \mathrm{~S}$ at $40^{\circ} \mathrm{C}$ in water) ${ }^{28}$. Also, anion exchange reactions (with TeTOP at the temperature range of $220-300^{\circ} \mathrm{C}$ ) have been shown to be effective for converting selenides to tellurides ${ }^{29}$. For the anion exchange reactions with TeTOP at high temperatures ${ }^{27,29}$, the chemical driving force was attributed to the fact that the $\mathrm{Te}=\mathrm{P}$ bond $\left(\sim 218 \mathrm{~kJ} \mathrm{~mol}^{-1}\right)$ is weaker than the $\mathrm{S}=\mathrm{P}$ $\left(\sim 402 \mathrm{~kJ} \mathrm{~mol}^{-1}\right)$ and $\mathrm{Se}=\mathrm{P}\left(\sim 314 \mathrm{~kJ} \mathrm{~mol}^{-1}\right)$ bonds (based on tri$n$-butylphosphine chalcogenide instead of ETOP $)^{37}$. For the anion exchange reactions in water ${ }^{28}$, they were reasoned to be influenced by the fact that the binding energy $\left(\sim 92 \mathrm{~kJ} \mathrm{~mol}^{-1}\right)$ of the Cd-Te bond is smaller than that $\left(\sim 157 \mathrm{~kJ} \mathrm{~mol}^{-1}\right)$ of the Cd-S bond ${ }^{38}$.

Here, we report the synthesis of colloidal alloy CdTeSe MSC399 and describe our argument for the formation pathway, as illustrated in Fig. 1. The MSCs obtained exhibit a single persistent and sharp absorption singlet peaking at $399 \mathrm{~nm}$. Separately, two induction period samples of binary CdTe and CdSe are prepared at relatively high temperatures $\left(120-140{ }^{\circ} \mathrm{C}\right)^{22-24}$; they are mixed afterwards at room temperature. The resulting mixture, together with the two binary samples, are characterized by optical absorption spectroscopy, electrospray ionization mass spectrometry (ESI-MS), and ${ }^{113} \mathrm{Cd}$ nuclear magnetic resonance (NMR). Evidently, alloy CdTeSe MSC-399 in a single ensemble form evolves from the mixture, without the emergence of otherbandgap MSCs and QDs. To explore the formation pathway of MSC-399, some control experiments were carried out, together with synchrotron-based in situ small angle X-ray scattering (SAXS). Critically, SAXS suggests that the two binary samples and their mixture all have similar particle sizes. For the formation of CdTeSe MSC-399 from the two binary induction period samples (IPS) (Equation (1)), we propose that it is the substitution reactions (Equations (2) and (3)), predominating over the addition reaction of the CdTe and CdSe precursor compounds (PCs), that result in the production of the precursor compound (CdTeSe PC) for CdTeSe MSC-399 (Equation (4)).

$$
\begin{gathered}
\text { CdTe IPS + CdSe IPS } \rightarrow \text { CdTeSe MSC }-399 \\
\text { CdTe PC }+ \text { CdSe M } / \mathrm{F} \rightarrow \text { CdTeSe PC }
\end{gathered}
$$

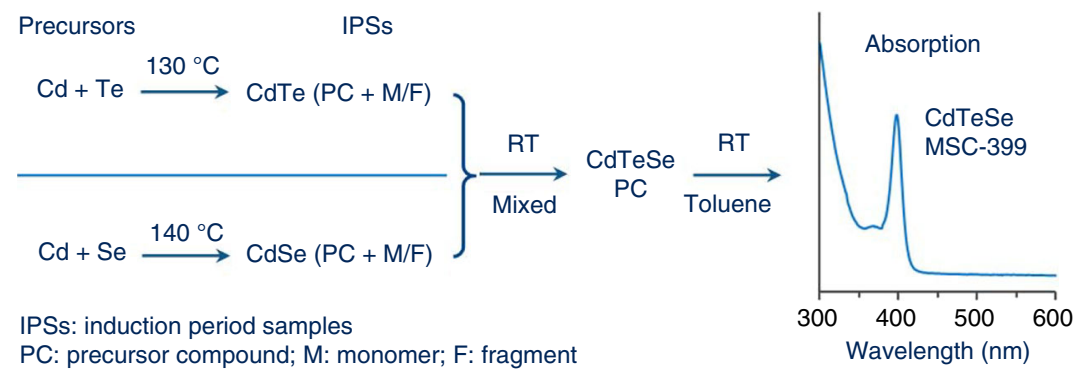

Fig. 1 Schematic outlining the synthesis of alloy CdTeSe MSC-399 and formation hypothesis. The alloy MSCs are prepared at room temperature from the mixture of the two induction period samples (IPSs) of binary CdTe and CdSe (Equation (1)). Cd and Te or Cd and Se precursors are independently mixed at room temperature and heated at $\sim 130$ or $\sim 140{ }^{\circ} \mathrm{C}$ to prepare the IPS, which has the precursor compounds (PCs) of CdTe MSC-371 or CdSe MSC-415, respectively, together with corresponding monomers $(M)$ and fragments $(F)$. The binary samples are mixed at room temperature for a period of time to produce MSC-399 via its precursor compound (CdTeSe PC, (Equation (4)), which is proposed to form via substitution reactions of the CdTe PC with the CdSe monomers and fragments (Equation (2)), and of the CdSe PC with the CdTe monomers and fragments (Equation (3)) 

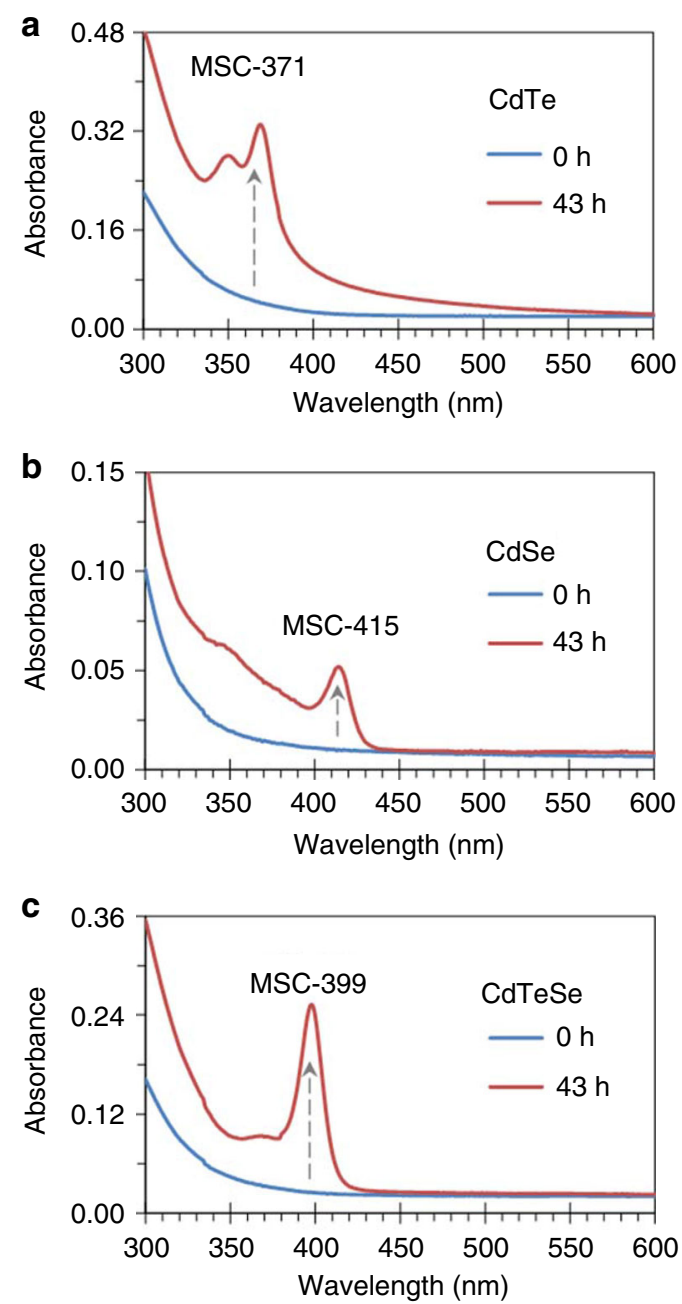

Fig. 2 Evolution of binary and ternary alloy MSCs. Optical absorption spectra of the induction period samples of binary $\mathrm{CdTe}(\mathbf{a})$ and $\mathrm{CdSe}(\mathbf{b})$, and their mixture (c) are collected before (blue traces) and after a 43-h incubation (red traces) at room temperature. The CdTe and CdSe samples were heated at 130 and $140{ }^{\circ} \mathrm{C}$ for $30 \mathrm{~min}$, respectively; they were cooled to room temperature and mixed with equal volumes. An aliquot of each sample $(30 \mu \mathrm{L})$ was dispersed in toluene $(3.0 \mathrm{~mL})$ for the measurement. During incubation, evidently, CdTe MSC-371 and CdSe MSC-415 evolved from the corresponding binary samples, while only CdTeSe MSC-399 evolved from their mixture

$$
\begin{gathered}
\text { CdSe PC }+ \text { CdTe M } / F \rightarrow \text { CdTeSe PC } \\
\text { CdTeSe PC } \rightarrow \text { CdTeSe MSC - } 399
\end{gathered}
$$

CdTe PC and CdSe PC represent the molecules that are able to transform to CdTe MSC-371 and CdSe MSC-415, respectively. $\mathrm{CdTe} \mathrm{M} / \mathrm{F}$ and $\mathrm{CdSe} \mathrm{M} / \mathrm{F}$ are symbolic of the binary monomers/ fragments, which are produced in the two corresponding binary CdTe IPS and CdSe IPS, respectively. The present study introduces a room-temperature approach to producing alloy MSCs in a single ensemble form without the co-production of otherbandgap MSCs and QDs, and provides insight into their probable formation pathways.

\section{Results}

Evolution of alloy CdTeSe MSC-399. Figure 2 shows the optical absorption spectra of two induction period samples of binary $\mathrm{CdTe}$ (a) and CdSe (b), together with their mixture (c) at room temperature before (blue traces) and after $43-\mathrm{h}$ incubation (red traces). The CdTe sample (a) was obtained after the $\mathrm{Cd}$ and $\mathrm{Te}$ precursors were mixed at room temperature and heated at $130{ }^{\circ} \mathrm{C}$ for $30 \mathrm{~min}^{23,24}$, while the $\mathrm{Cd}$ and Se precursors were mixed at room temperature and heated at $140{ }^{\circ} \mathrm{C}$ for $30 \mathrm{~min}$ to produce the CdSe sample $(b)^{25}$. The two binary samples were mixed in equal volumes at room temperature $(c)$. An aliquot $(30 \mu \mathrm{L})$ of each of the three samples before and after incubation was dispersed in toluene $(3.0 \mathrm{~mL})$, and its absorption spectrum was collected.

The blue traces illustrate that the binary samples and their mixture are transparent in optical absorption. The three featureless spectra suggest the absence of MSCs in these samples. The red traces of the incubated samples, exhibiting characteristically narrow absorption peaks, demonstrate the presence of CdTe MSC-371 (a), CdSe MSC-415 (b), and probable CdTeSe MSC-399 (c). The presence of the binary MSCs indicates the presence of their corresponding precursor compounds (PCs) in the two binary samples, together with monomers and fragments, and that the CdTe PC $\rightarrow$ MSC-371 and CdSe PC $\rightarrow$ MSC-415 transformations took place during incubation at room temperature ${ }^{16,21-26}$.

For the mixture of the binary samples after incubation, only the $399 \mathrm{~nm}$ peak is observed (red trace in Fig. 2c). Accordingly, we attribute it to a ternary CdTeSe system and label it MSC-399. The presence of MSCs is further supported by the persistent peak position at $399 \mathrm{~nm}$ as seen in Supplementary Figs. 1 to 3 . Importantly, neither CdTe MSC-371 nor CdSe MSC-415 is present in the incubated mixture. Accordingly, during the mixture incubation, the CdTe PC $\rightarrow$ MSC-371 and CdSe $\mathrm{PC} \rightarrow$ MSC-415 transformations apparently do not occur ${ }^{16,23,25}$, in accordance with the relevant process of the substitution reactions described by Equations (2) and (3), respectively. Hence, we conclude that during the mixture incubation, the reactions indicated by Equations (2) to (4) take place probably.

For the synthesis of MSC-399, we explore the effects of the temperatures used when heating the two binary CdTe and CdSe samples (Supplementary Fig. 1), together with the heating periods (Supplementary Fig. 2) and their mixing volume ratios (Supplementary Fig. 3). The substitution reactions described by Equations (2) and (3) are able to explain well the experimental results obtained. Remarkably, for the samples extracted from the reaction batch consisting of the $\mathrm{Cd}, \mathrm{Te}$, and Se precursors together (such as during the temperature range of $120-150{ }^{\circ} \mathrm{C}$ ), apparently, no MSC-399 is present (Supplementary Fig. 4). We argue that this absence is due to the fact that the formation of Cd-Te bonds takes place at a lower temperature than that needed for Cd-Se bonds (in a reaction batch) $2,6,7,23-25$. Furthermore, the formation of CdTe QDs (taking place at $\sim 150{ }^{\circ} \mathrm{C}$ (trace 4 in Supplementary Fig. 4)) consumed the CdTe PC produced (via the PC fragmentation to QD pathway $)^{15}$. Thus, the formation of CdTeSe PC was hindered.

ESI-MS of binary samples and their mixture. ESI-MS has been applied to investigate the formation of $\mathrm{M}-\mathrm{E}$ covalent bonds in the first-step sample of the selective two-step approach; the first step is controlled to be within the induction period $21,24,25$. For those fragments detected, they do not seem to display any surface ligands. Furthermore, the fragment interval between two nearestneighbor peaks is $1 \mathrm{Da}$, indicating that the bare fragments are mono-charged $^{39,40}$.

Figure 3 displays the ESI-MS spectra within the $m / z$ range of 1200-1700 Da, for the samples of binary CdTe (trace 1) and CdSe (trace 2) (without room temperature incubation), together with their room temperature mixture (trace 3 ) with equal volumes and 30 min incubation. The CdTe and CdSe samples were heated at 


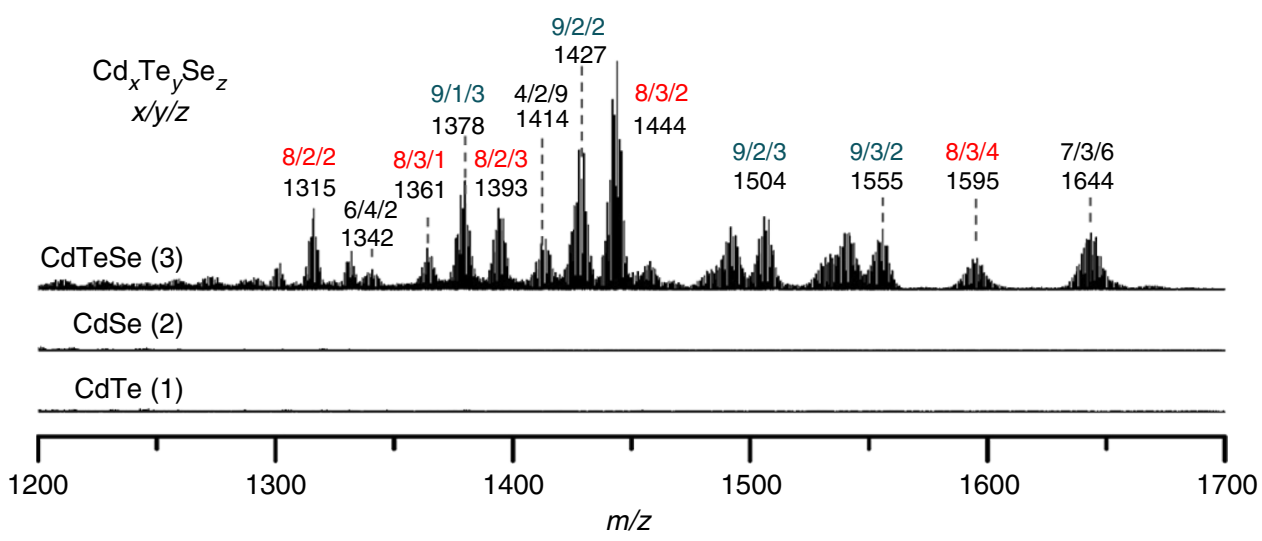

Fig. $3 \mathrm{ESI}-\mathrm{MS}$ spectra of two binary samples and their mixture. Both the binary $\mathrm{CdTe}$ (trace 1) and $\mathrm{CdSe}$ (trace 2 ) samples were heated at $120^{\circ} \mathrm{C}$ for $30 \mathrm{~min}$; they were mixed at room temperature with equal volumes; the resulting mixture (trace 3 ) was incubated for 30 min. The $\mathrm{Cd}_{x} \mathrm{Te}_{y} \mathrm{Se}_{z}$ fragments (trace 3 ) suggest the formation of Te-Cd-Se covalent bonds in the mixture, which is in agreement with the substitution reactions described by Equations (2) and (3)

$120^{\circ} \mathrm{C}$ for $30 \mathrm{~min}$. Supplementary Fig. 7 presents the same ESIMS collection but in the $\mathrm{m} / z$ range of $950-1250 \mathrm{Da}$. For the fragments detected from the mixture (trace 3), some of them are unambiguously different from those monitored from the two binary samples (traces 1 and 2). We tried to assign these fragments to $\mathrm{Cd}_{x} \mathrm{Te}_{y} \mathrm{Se}_{z}$ (where $\mathrm{x}, \mathrm{y}$, and $\mathrm{z}$ are integer values), based on the $\mathrm{Cd}$, Te, $\mathrm{Se}, \mathrm{Cd}_{1} \mathrm{Te}_{1}, \mathrm{Cd}_{1} \mathrm{Se}_{1}$, and $\mathrm{Cd}_{1} \mathrm{Te}_{1} \mathrm{Se}_{1}$ isotopic patterns and peak positions (Supplementary Fig. 8 and Supplementary Note 1$)^{20,23,24}$. Supplementary Fig. 9 presents the expanded views of the $\mathrm{Cd}_{x} \mathrm{Te}_{y} \mathrm{Se}_{z}$ fragments assigned in the $\mathrm{m} / z$ range of 950-1700 Da. Based on the formula of $x+y+z$ $(=9-16)$, it seems that the two 11-atom species, $\mathrm{Cd}_{7} \mathrm{Te}_{1} \mathrm{Se}_{3}$ and $\mathrm{Cd}_{6} \mathrm{Te}_{2} \mathrm{Se}_{3}$, were produced most often, among the fragmented species consisting of 9-16 atoms. The formation of the monocharged fragments could be related to surface ligand detachment; ${ }^{21,24,25,41,42}$ intriguingly, the charge of the fragments presented here is different from the overall charge calculated based on $\mathrm{Cd}^{2+}, \mathrm{Te}^{2-}$, and $\mathrm{Se}^{2-}$.

For the binary samples of $\mathrm{CdTe} 130^{\circ} \mathrm{C} / 30 \mathrm{~min}$ and $\mathrm{CdSe} 140^{\circ}$ $\mathrm{C} / 30 \mathrm{~min}$, together with their mixture used for Fig. 2, Supplementary Fig. 10 presents their ESI-MS spectra. Again, some of the fragmented species detected from the mixture differ from those from the two binary samples. Supplementary Fig. 11 highlights the comparison of the fragments obtained, for the CdTe $120^{\circ} \mathrm{C} /$ $30 \mathrm{~min}$ vs $130^{\circ} \mathrm{C} / 30 \mathrm{~min}$ samples, the $\mathrm{CdSe} 120^{\circ} \mathrm{C} / 30 \mathrm{~min}$ vs $140^{\circ} \mathrm{C} / 30 \mathrm{~min}$ samples, and their corresponding mixtures. It seems that, for the two binary samples, more fragments are obtained from the higher temperature samples. Accordingly, Fig. 3 and Supplementary Fig. 10 provide indirect but convincing evidence for the formation of Te-Cd-Se covalent bonds in the mixture of the two binary samples. The ESI-MS study is thus supportive of the relevance of the substitution reactions represented by Equations (2) and (3), and that the substitution reactions readily take place at room temperature (within $30 \mathrm{~min}$ ). The ESI-MS study is in agreement with the statement that MSC399 has a ternary nature.

${ }^{113}$ Cd NMR of binary samples and their mixture. In view of its sensitivity to the local environment, ${ }^{113} \mathrm{Cd}$ NMR spectroscopy has been used to follow the formation of $\mathrm{Cd}-\mathrm{Te}$ covalent bonds in induction period samples ${ }^{24}$. Also, it has provided important information regarding the formation of alloy CdTeSe MSC-520 and CdTeSe QDs 2,7 . We now present the NMR investigation of the formation of $\mathrm{Te}-\mathrm{Cd}-\mathrm{Se}$ covalent bonds in the mixture of two binary samples of CdTe and CdSe.

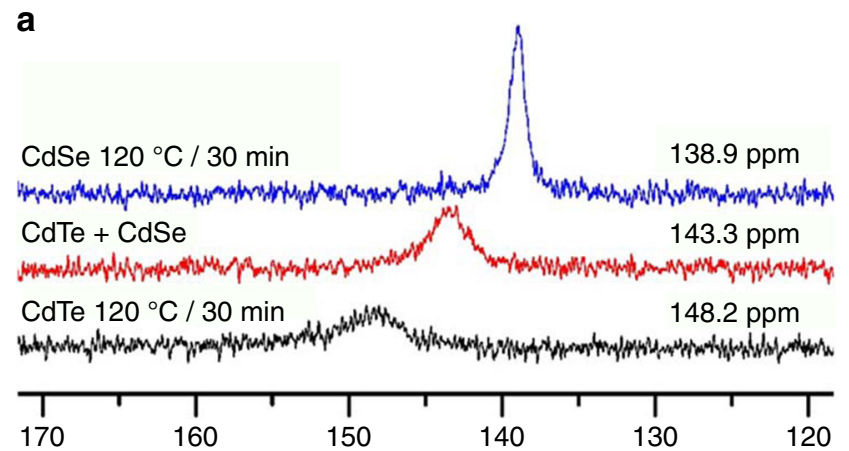

b

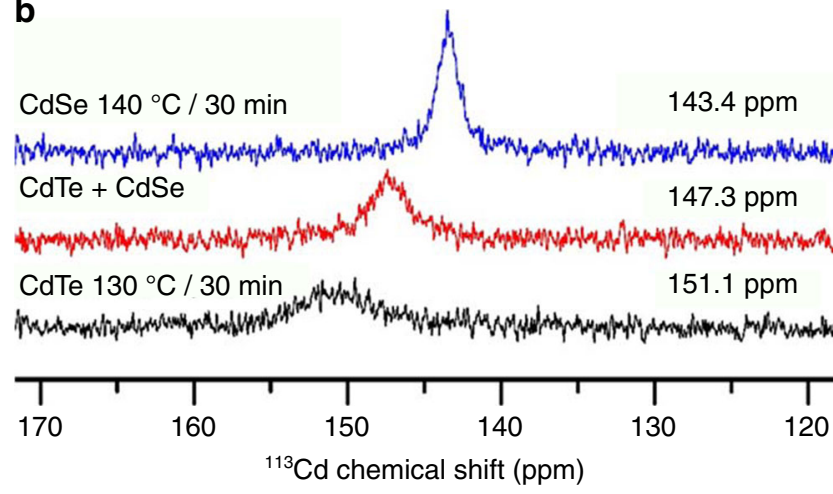

Fig. $4{ }^{113} \mathrm{Cd}$ NMR spectra of two sets of binary samples and their mixtures. a Both binary samples for CdTe (black trace) and CdSe (blue trace) were reacted at $120^{\circ} \mathrm{C}$ for $30 \mathrm{~min}$, and the mixture (red trace) was obtained by mixing the two binary samples with equal volumes at room temperature for 15 min incubation. b CdTe (black trace) and CdSe (blue trace) samples were reacted for $30 \mathrm{~min}$ at 130 and $140{ }^{\circ} \mathrm{C}$, respectively, and their mixture (red trace) was obtained with equal volumes at room temperature for $15 \mathrm{~min}$ incubation. The binary samples were prepared as indicated. The ${ }^{113} \mathrm{Cd}$ resonance signals of the two mixtures (red traces), locating between those from the binary samples, are in agreement with the substitution reactions described by Equations (2) and (3)

Figure 4 shows the ${ }^{113} \mathrm{Cd}$ NMR spectra collected from two CdTe samples (black traces), two CdSe samples (blue traces), and the corresponding two mixtures (red traces, with $15 \mathrm{~min}$ incubation at room temperature). $\mathrm{Cd}\left(\mathrm{ClO}_{4}\right)_{2}$ is used as a chemical shift reference. The binary samples in Fig. $4 \mathrm{a}$ are heated at $120^{\circ} \mathrm{C}$ for $30 \mathrm{~min}$, whereas the samples in the Fig. $4 \mathrm{~b}$ are heated for 

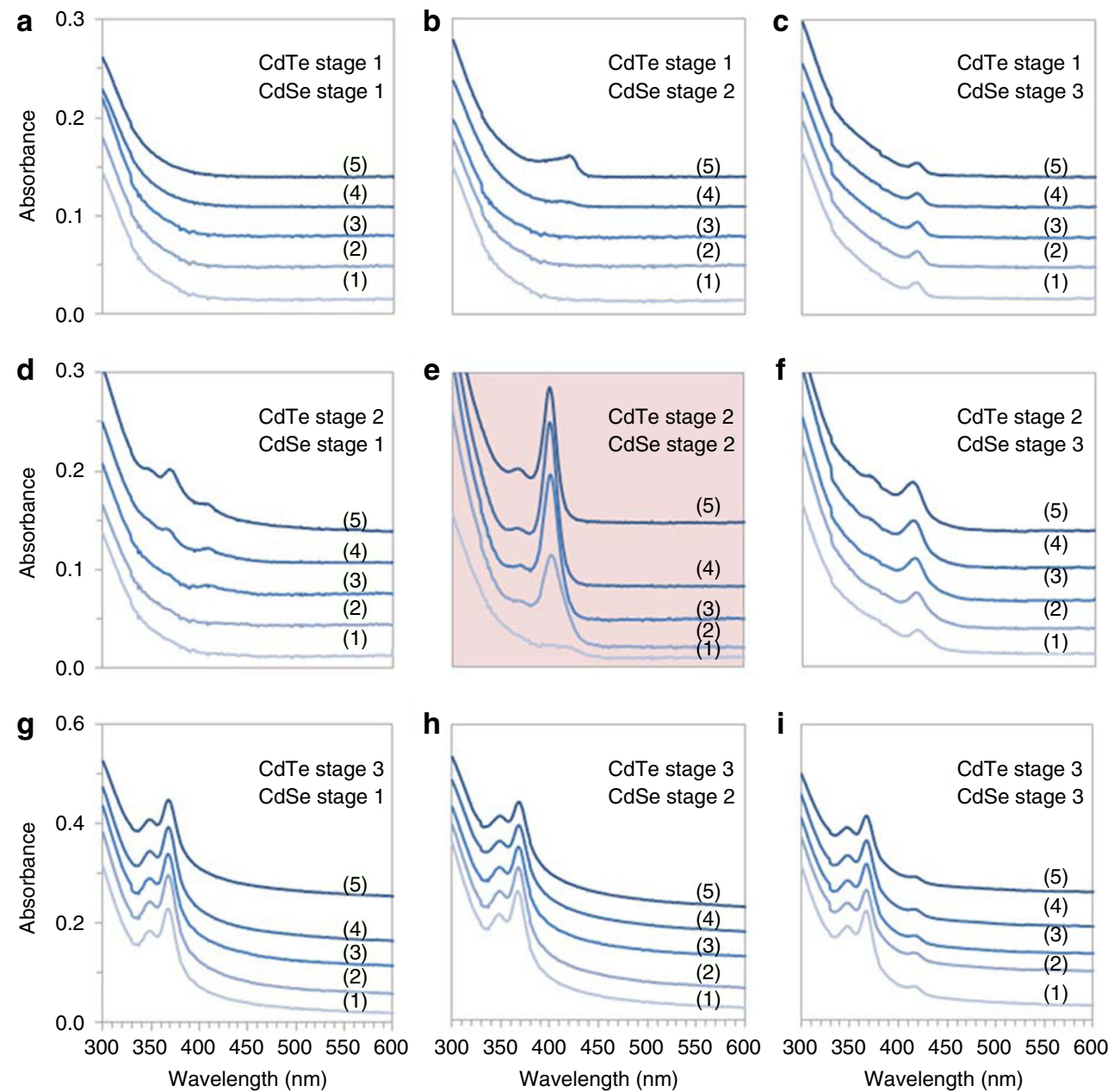

Fig. 5 Optical absorption spectra of nine mixtures of $\mathrm{CdTe}$ and $\mathrm{CdSe}$. There are nine mixtures prepared by mixing $\mathrm{CdTe}$ and $\mathrm{CdSe}$ samples in Stages 1 and 1 (a), 1 and 2 (b), 1 and 3 (c), 2 and 1 (d), 2 and 2 (e), 2 and 3 (f), 3 and 1 (g), 3 and 2 (h), and 3 and 3 (i), at room temperature with equal volumes. The mixtures were incubated for (1) $0 \mathrm{~min},(2) 1 \mathrm{~h},(3) 2 \mathrm{~h},(4) 4 \mathrm{~h}$, and (5) $8 \mathrm{~h}$. Each of the mixtures (30 $\mu \mathrm{L})$ was dispersed in toluene (3.0 mL). Interestingly, MSC-399 was well evolved only from the mixture shown in Part e with the mixture of Stage $2 \mathrm{CdTe}$ and CdSe. Stage 1 means when two precursors of Cd and $\mathrm{Te}$ or $\mathrm{Cd}$ and Se were put together at room temperature; after Stage 1 samples were heated for $30 \mathrm{~min}$ at $130^{\circ} \mathrm{C}$ for $\mathrm{CdTe}$ or at $140{ }^{\circ} \mathrm{C}$ for $\mathrm{CdSe}$, they are referred as Stage 2 (induction period) samples. When the Stage 2 samples were incubated at room temperature for $24 \mathrm{~h}$, they are called Stage 3 samples (with the presence of CdTe MSC-371 or CdSe MSC-415)

$30 \mathrm{~min}$ at $130^{\circ} \mathrm{C}$ for $\mathrm{CdTe}$ and at $140{ }^{\circ} \mathrm{C}$ for CdSe. Again, the two mixtures were prepared via the equal volume mixing of the two sets of the binary samples. The samples $(0.3 \mathrm{~mL})$ were diluted with toluene- $d_{8}(0.3 \mathrm{~mL})$. Supplementary Table 1 provides the details for the data collection.

For the two spectra displayed in red, the ${ }^{113} \mathrm{Cd}$ resonance signals obtained are located between those of the corresponding binary samples. Accordingly, the two resonance signals are consistent with the formation of $\mathrm{Te}-\mathrm{Cd}-\mathrm{Se}$ covalent bonds via the substitution reactions denoted by Equations (2) and (3). Interestingly, the ${ }^{113} \mathrm{Cd}$ resonance signals shift down-field, from the CdTe $120^{\circ} \mathrm{C}$ sample $(148.2 \mathrm{ppm})$ to $130^{\circ} \mathrm{C}$ sample $(151.1 \mathrm{ppm})$, and from the CdSe $120^{\circ} \mathrm{C}$ sample $(138.9 \mathrm{ppm})$ to $140{ }^{\circ} \mathrm{C}$ sample $(143.4 \mathrm{ppm})$. Such shifts are attributed to the formation of more $\mathrm{Cd}-\mathrm{Te}$ or $\mathrm{Cd}-\mathrm{Se}$ bonds $^{24}$. At the same time, there is a certain degree of down-field shifting for the ${ }^{113} \mathrm{Cd}$ resonance signals from the mixture depicted in Fig. 4a (143.3 ppm) to the mixture of Fig. $4 \mathrm{~b}$ (147.3 ppm). Accordingly, the concentration of $\mathrm{Te}-\mathrm{Cd}-\mathrm{Se}$ bonds in the Fig. 4a mixture appears to be smaller than that in the Fig. $4 \mathrm{~b}$ mixture; this difference is consistent with the results shown in Supplementary Figs. 1 and 11. It is of help to point out that the NMR measurements are performed without sample purification, as we did for the CdTe binary system ${ }^{24}$. The presence of one single ${ }^{113} \mathrm{Cd}$ resonance signal was argued to be related to fast chemical exchange processes in one sample ${ }^{24}$. We anticipate the same for the present study.

To further endorse our comprehension of the red trace shown in Fig. 4b, which is indicative of the formation of the alloy PC (from substitution reactions described by Equations (2) and (3)), we design a background experiment (Supplementary Fig. 12), in which two binary samples of CdTe and CdSe are placed closely enough but without mixing. Supplementary Fig. 12 illustrates the experimental arrangement and displays the NMR spectrum collected from the samples of CdTe $130^{\circ} \mathrm{C} / 30 \mathrm{~min}$ and $\mathrm{CdSe}$ $140^{\circ} \mathrm{C} / 30 \mathrm{~min}$. This spectrum in Supplementary Fig. 12 has two discernible ${ }^{113} \mathrm{Cd}$ resonance signals at $\sim 151.8$ and $\sim 143.0 \mathrm{ppm}$ and is understandably different from the corresponding spectrum (red trace) in Fig. $4 \mathrm{~b}$. The chemical shift values are similar to those of the CdTe (black trace) and CdSe (blue trace) spectra shown in Fig. 4b, respectively. This Supplementary Fig. 12 result provides further compelling evidence which supports the substitution reactions designated by Equations (2) and (3). 

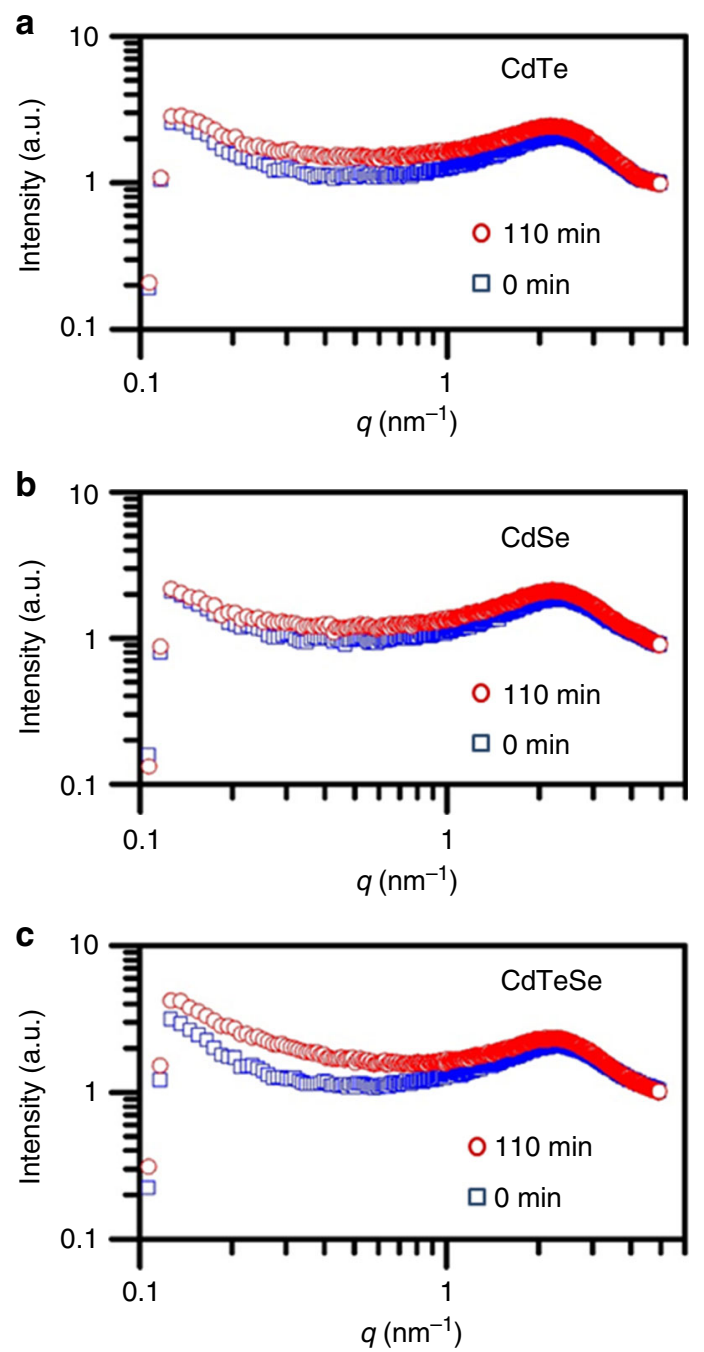

Fig. 6 In situ SAXS profiles for two binary samples and their mixture. The binary $\mathrm{CdTe}(\mathbf{a})$ and $\mathrm{CdSe}(\mathbf{b})$ samples were reacted at 130 and $140{ }^{\circ} \mathrm{C}$, respectively, for $30 \mathrm{~min}$ and the mixture (c) was obtained at room temperature by mixing the binary samples with equal volumes. The SAXS data were collected each $10 \mathrm{~min}$ up to $110 \mathrm{~min}$, with the spectra of 0 (blue squares) and $110 \mathrm{~min}$ (red circles) presented here. The SAXS study illustrates that the size of the three samples are clearly similar (as demonstrated by Supplementary Table 2 and Supplementary Fig. 16)

\section{Discussion}

With regard to the formation pathway of MSC-399, we first considered chalcogenide (E) anion exchange reactions. Supplementary Fig. 13 shows the optical absorption properties of the mixture of $\mathrm{CdTe}\left(130^{\circ} \mathrm{C} / 30 \mathrm{~min}\right)$ and SeTOP, as well as those of the mixture of $\mathrm{CdSe}\left(140^{\circ} \mathrm{C} / 30 \mathrm{~min}\right)$ and TeTOP. For the former mixture, CdTe MSC-371 is monitored (after $4 \mathrm{~h}$ of mixing), while any possible CdSe MSC-415 is sought from the latter mixture. Also, it is apparent that no CdTeSe MSC-399 evolves from the two mixtures. Accordingly, it seems reasonable that anion exchange reactions (with SeTOP or TeTOP) do not take place at room temperature. In addition to Supplementary Fig. 13, additional control experimental results are presented in Fig. 5, which addresses the possible formation of MSC-399 from mixtures made from binary samples at three stages.

The CdTe and CdSe samples used are categorized as Stages 1-3. When the two precursors of $\mathrm{Cd}$ and Te or $\mathrm{Cd}$ and Se were put together at room temperature (in oleylamine (OLA) to prepare one induction period sample), the resulting mixture is referred to as a Stage 1 sample. After the Stage 1 sample was heated for $30 \mathrm{~min}$ at $130{ }^{\circ} \mathrm{C}$ for $\mathrm{CdTe}$ or at $140{ }^{\circ} \mathrm{C}$ for CdSe, it is designated as a Stage 2 sample. When Stage 2 samples were incubated at room temperature for $24 \mathrm{~h}$ (during which CdTe MSC-371 and CdSe MSC-415 evolved), they are denoted as Stage 3 samples.

The ${ }^{113} \mathrm{Cd}$ NMR signals in Fig. $4 \mathrm{~b}$ (for Stage $2 \mathrm{CdTe}$, Stage 2 $\mathrm{CdSe}$, and the mixture of the two) are evidently different from those collected from Stage $1 \mathrm{CdTe}$, Stage $1 \mathrm{CdSe}$, and the mixture of the two, together with the $\mathrm{Cd}$ precursor $\mathrm{Cd}(\mathrm{OAc})_{2} / \mathrm{OLA}$ (Supplementary Fig. 14). The chemical shifts of the four ${ }^{113} \mathrm{Cd}$ NMR signals are similar (in the range of 138-140 ppm). For the three ${ }^{113} \mathrm{Cd}$ resonance signals illustrated in Fig. $4 \mathrm{~b}$, they are much broader than these four in Supplementary Fig. 14, together with down-field chemical shifts (143-151 ppm). CdTe PCs and CdSe PCs are not formed in Stage $1 \mathrm{CdTe}$ and Stage $1 \mathrm{CdSe}$, respectively ${ }^{24,25}$. By the way, a recent study documents that the CdSe binary PC of CdSe MSC-415 is white in colour with a composition of $2 \mathrm{Cd}$ to $1 \mathrm{Se}^{43}$; we argue that the cause for the enough amount of the PC formed is related to its own formation pathway in the induction period ${ }^{16,21-26}$. The conversion of SeTOP in an induction period (of CdSe QDs with the growth period of less than $5 \mathrm{~min}$ ) was documented to be around $20 \%{ }^{17}$. For the present study, the TeTOP and SeTOP conversion is estimated to be about $37 \%$ and $16 \%$, respectively, for Stage $2 \mathrm{CdTe}$ and Stage $2 \mathrm{CdSe}$ (Supplementary Fig. 15).

Thus, the ${ }^{113} \mathrm{Cd}$ NMR study (Fig. 4 and Supplementary Fig. 14) is in agreement with the absence and presence of the two binary PCs at relatively low and high temperatures, respectively. Accordingly, the ${ }^{113} \mathrm{Cd}$ resonance signal (red trace of Fig. $4 \mathrm{~b}$ ) is indicative of the formation of CdTeSe alloy PCs. Undoubtedly, these Stages 1-3 samples have different amounts of monomers, fragments, and PCs. Stage 1 samples have the Cd precursor and TeTOP or the Cd precursor and SeTOP, without the formation of $\mathrm{Cd}-\mathrm{Te}$ and $\mathrm{Cd}-\mathrm{Se}$ covalent bonds ${ }^{24,25}$. Stage 2 samples are induction period samples containing binary PCs and monomers and fragments. Stage 3 samples have undergone the PC $\rightarrow$ MSC transformation and thus have a smaller number of PCs ${ }^{16,21-26}$.

Figure 5 shows optical absorption spectra collected from nine mixtures made from CdTe and CdSe in equal volumes at room temperature, which provides further support regarding the substitution reactions signified by Equations (2) and (3). Intriguingly, the optimum formation of MSC-399 is found to be the mixture where CdTe Stage 2 and CdSe Stage 2 are mixed, as shown by Fig. 5e (highlighted in red). The strength of the $399 \mathrm{~nm}$ peak intensifies, as more MSC-399 is formed due to the PC $\rightarrow$ MSC transformation (occurring in the mixture up to $4 \mathrm{~h}$ incubation at room temperature).

In all other cases, MSC-399 is not observed. There are no absorption peaks seen in Sample a, while for the other samples, MSC-371 and/or MSC-415 characteristic absorption peaks are present. For the mixture (a) of CdTe Stage 1 and CdSe Stage 1, the absence of any MSC characteristic peaks can be attributed to the absence of $\mathrm{Cd}-\mathrm{Te}$ and $\mathrm{Cd}-\mathrm{Se}$ bonds, for interactions between the Cd, Te, and Se precursors at room temperature ${ }^{24,25}$. For the mixture (b) of CdTe Stage 1 and CdSe Stage 2, the emergence of a peak at $415 \mathrm{~nm}$ indicates the appearance of MSC-415; this is in agreement with that anion exchange reactions of TeTOP and CdSe PCs does not take place during incubation, but the CdSe PC $\rightarrow$ MSC-415 transformation does. For the mixture (c) of CdTe Stage 1 and CdSe Stage 3, a peak at $415 \mathrm{~nm}$ changes little over incubation; thus, TeTOP has no effect on CdSe MSC-415 either.

For the mixture (d) of CdTe Stage 2 and CdSe Stage 1, the gradual appearance of a peak at $371 \mathrm{~nm}$ indicates the presence of CdTe MSC-371; this is consistent with the absence of anion exchange reactions of CdTe PCs and SeTOP, and with the 
presence of the CdTe PC $\rightarrow$ MSC-371 transformation during incubation. For the mixture (f) of CdTe Stage 2 and CdSe Stage 3, a peak at $371 \mathrm{~nm}$ seems to emerge gradually, which indicates that during incubation, the CdTe PC $\rightarrow$ MSC-371 transition takes place and is not significantly affected by the presence of the CdSe sample. The absence of significant change for the $415 \mathrm{~nm}$ peak seems to suggest that MSC-415 is not affected by the CdTe PC $\rightarrow$ MSC-371 transition.

For the mixture $(\mathrm{g})$ of CdTe Stage 3 and CdSe Stage 1, the $371 \mathrm{~nm}$ peak remains essentially constant; thus, MSC-371 appears unaffected by SeTOP. For the mixture (h) of CdTe Stage 3 and CdSe Stage 2, the strength of the $371 \mathrm{~nm}$ peak is clearly constant; thus, MSC-371 appears unaffected by CdSe PCs. No $415 \mathrm{~nm}$ peak evolves, suggesting that the CdSe PC $\rightarrow$ MSC-415 transition is slowed down somehow by the presence of the CdTe sample. Finally, for the mixture (i) of CdTe Stage 3 and CdSe Stage 3, the two peaks at 371 and $415 \mathrm{~nm}$ are clearly identifiable and constant, indicating that MSC-371 and MSC-415 do not substantially interfere with each other. The formation of the binary MSCs consumes the corresponding PCs; thus, CdTeSe PCs do not form and thus no MSC-399 evolves.

To further explore the formation pathway of MSC-399, synchrotron-based in situ SAXS is performed. SAXS has been widely used to provide valuable information such as the size of targets of interest $\mathrm{t}^{24,44,45}$. For the present study, SAXS provides valuable information on the size of induction period samples of binary $\mathrm{CdTe}$ and CdSe, together with their room temperature mixtures. The SAXS data are collected each $10 \mathrm{~min}$ up to $110 \mathrm{~min}$ (Supplementary Fig. 16). With the 0 (blue squares) and $110 \mathrm{~min}$ (red circles) spectra presented only, Fig. 6 displays the SAXS profiles collected at room temperature of CdTe (a), CdSe (b), and their mixture (c). The two binary samples are heated for $30 \mathrm{~min}$ at $130^{\circ} \mathrm{C}$ for $\mathrm{CdTe}$ and at $140^{\circ} \mathrm{C}$ for CdSe. The peaks at approximately $2 \mathrm{~nm}^{-1}$ are attributed to inter-particle scattering from the species in CdTe (a) and CdSe (b) and the mixture (c).

Based on the SAXS profiles obtained from the two binary samples and their mixture (shown in Fig. 6), Supplementary Table 2 summarizes the overall sizes. Up to $110 \mathrm{~min}$, there is a steady increase in the overall size from 0.8 to $1.0 \mathrm{~nm}$ for CdTe, 0.8 to $0.9 \mathrm{~nm}$ for CdSe, and 0.8 to $1.0 \mathrm{~nm}$ for the mixture. Unambiguously, the sizes obtained from the CdTe, CdSe, and mixture samples are quite similar, and the trend with time is similar too. Supplementary Fig. 17 presents the overall size estimated for the binary samples of $\mathrm{CdTe}, \mathrm{CdSe}$, and their mixture based on the measurements up to $110 \mathrm{~min}$. Supplementary Fig. 18 presents the corresponding absorption properties of the Fig. 6 samples along with the SAXS data collection. The evolution of MSC-371 and MSC-399 is clearly demonstrated in Parts a and c of Supplementary Fig. 18, respectively.

In general, the SAXS study shows that no larger species formed in the mixture of the CdTe and CdSe samples. Critically, the mixture has a similar size to those of the two binary samples. Therefore, one CdTeSe PC should not be resulted from an addition reaction of one CdTe PC with one CdSe PC, but probably from the substitution reactions symbolized by Equations (2) and (3).

In conclusion, we have effectively synthesized alloy CdTeSe MSC-399 and have explored its formation pathway. Two induction period samples (IPS) of binary CdTe and CdSe are independently prepared at elevated temperatures, and are mixed at room temperature (Equation (1)). The resulting MSC-399 is in a single-ensemble form without the presence of other-bandgap MSCs and QDs. Intriguingly, for a conventional approach consisting of $\mathrm{Cd}$, Te and Se precursors, the reaction is not able to produce the CdTeSe MSCs (Supplementary Fig. 4). To explore the formation pathway, a combination of characterization tools is applied, including optical absorption spectroscopy, ESI-MS, ${ }^{113} \mathrm{Cd}$ NMR, and synchrotron-based in situ SAXS. Only one optical absorption peak at $399 \mathrm{~nm}$ evolves from the binary mixture after incubation (Fig. 2), suggesting that the CdTe PC $\rightarrow$ MSC-371 and CdSe PC $\rightarrow$ MSC-415 transformation do not take place, but the substitution reactions (Equations (2) and (3)) do, together with the CdTeSe PC $\rightarrow$ MSC-399 transformation (Equation (4)). Our ESI-MS study (Fig. 3) illustrates that the substitution reactions to CdTeSe PC occur quickly; $\mathrm{Cd}_{x} \mathrm{Te}_{y} \mathrm{Se}_{z}$ fragment species are detected when two induction period samples of binary CdTe and CdSe are mixed at room temperature for $30 \mathrm{~min}$. Furthermore, the formation of $\mathrm{Te}-\mathrm{Cd}-\mathrm{Se}$ covalent bonds at room temperature is supported by ${ }^{113} \mathrm{Cd}$ NMR (Fig. 4); the ${ }^{113} \mathrm{Cd}$ resonance signal of a mixture of two induction period samples of binary CdTe and CdSe is located between those of the two binary samples. Moreover, the size of a binary mixture obtained is similar to those of corresponding binary CdTe and CdSe, as suggested by SAXS (Fig. 6). Therefore, it seems reasonable to hypothesize that the formation of CdTeSe MSC-399 at room temperature does not come from the addition of CdTe MSC-371 and CdSe MSC-415, but is via its own precursor compound CdTeSe PC-399 (Equation (4)). The formation of CdTeSe PC-399 at room temperature is neither via partial anion exchange reactions (of CdTe PC + SeTOP or CdSe PC + TeTOP, Fig. 5 and Supplementary Fig. 13), nor via the addition reaction of CdTe PC-371 and CdSe PC-415, but is via the substitution reactions (Equations (2) and (3)). Exploring the chemistry at the nanoscale, the present effort on CdTeSe MSC-399 narrows the knowledge gap on the synthesis and formation pathway of ternary MSCs at room temperature. It is probable that the room temperature approach to ternary CdTeSe MSC-399 developed and the formation pathway proposed in present study is applicable to other ternary semiconductors MSCs such as CdTeS. We are actively exploring the applicability of the approach, together with the structure of binary MSCs using Random Structure Searching methods with DFT calculations ${ }^{46}$. The presence of a protic agent such as methanol or a primary amine has been documented to accelerate the binary PC to binary MSC transformation ${ }^{16,21-26,47-49}$. For the formation of CdSe MSC-415 (from CdSe QDs) via the presence of a primary amine ${ }^{49}$, the present study enables us to comprehend the pathway to be probably via CdSe PC-415 from the QD fragmentation; however, in-depth physical insight for the process still requires more efforts (such as the exploration of activation energy). This subject will be our forthcoming study, which will be also extended to alloy MSCs. Given the complexity of the subject, it is impossible to extract complete mechanistic insight from one study. Even so, the present study is one solid piece (in a very large puzzle), providing the formation pathway of the alloy MSCs and making the synthesis less empirical. We believe that the field of colloidal nanocrystals is transforming, as it must, from an empirical art to science, similar to the advance of organic chemistry ${ }^{50-55}$.

\section{Methods}

Chemicals. Cadmium acetate dihydrate $\left(\mathrm{Cd}(\mathrm{OAc})_{2} \bullet 2 \mathrm{H}_{2} \mathrm{O} 99.999 \%\right.$, Alfa Aesar), tellurium powder (99.99\%, Alfa Aesar), selenium powder (99.99\%, Alfa Aesar), oleylamine (OLA, $90 \%$, Aldrich), tri- $n$-octylphosphine (TOP, $90 \%$, Aldrich), octylamine (OTA, 99\%, Aldrich), ethylacetate (99.5\%, Tianjin Zhiyuan Chemical) and nitrogen gas $\left(\mathrm{N}_{2}, 99.99 \%\right.$, Chengdu Taiyu gas Co. Ltd.) were used as received without further purification unless stated otherwise. Toluene (AR grade, Chengdu Kelong Chemical) was distilled and further dried with $\mathrm{MgSO}_{4}(99 \%$, Tianjin Zhiyuan Chemical). Oleylamine (OLA) was stored in a freezer, while the other chemicals were stored under an ambient environment.

Cd precursor preparation. $\mathrm{Cd}(\mathrm{OAc})_{2} \cdot 2 \mathrm{H}_{2} \mathrm{O}(0.160 \mathrm{~g}, 0.6 \mathrm{mmol})$ and $\mathrm{OLA}$ $(3.000 \mathrm{~g})$ were added into a $50 \mathrm{~mL}$ three-necked flask at room temperature, and the flask was evacuated and then purged with $\mathrm{N}_{2}$ gas; this procedure was repeated three times until no bubbles were observed under vacuum. Then, the mixture was heated 
up to $80^{\circ} \mathrm{C}$ under a $\mathrm{N}_{2}$ atmosphere, followed by evacuation for an hour until no bubbles were apparent. Under a $\mathrm{N}_{2}$ atmosphere, the mixture was heated up to $120^{\circ}$ $\mathrm{C}$, and was kept under vacuum for $1 \mathrm{~h}$. In this way, our $\mathrm{Cd}$ precursor was obtained in the form of a clear, light yellow solution.

Preparation of Te and Se precursors. The Te and Se precursors were tri- $n$ octylphosphine telluride (TeTOP) and tri- $n$-octylphosphine selenide (SeTOP), respectively, with a feed molar ratio of $4 \mathrm{TOP}$ to $1 \mathrm{E}(\mathrm{E}=\mathrm{Te}$ or Se). Tellurium powder $(0.019 \mathrm{~g}, 0.15 \mathrm{mmol})$ or Selenium powder $(0.012 \mathrm{~g}, 0.15 \mathrm{mmol})$ and TOP $(0.247 \mathrm{~g}, 0.6 \mathrm{mmol})$ was added into a $25 \mathrm{~mL}$ three-necked reaction flask at room temperature. The mixture was degassed under vacuum and purged with $\mathrm{N}_{2}$ gas; this process was repeated three times. Under a $\mathrm{N}_{2}$ environment, the Te mixture was heated to $300^{\circ} \mathrm{C}$ for $30 \mathrm{~min}$, while the Se mixture was heated to $40^{\circ} \mathrm{C}$ for $10 \mathrm{~min}$. When the two clear solutions were cooled to room temperature, OLA $(1.574 \mathrm{~g}$ for TeTOP and $1.581 \mathrm{~g}$ for SeTOP) was added (in order to reach a total weight of $5.00 \mathrm{~g}$ for the binary sample preparation).

Preparation of CdTe and CdSe samples and their mixtures. Reactions were carried out with a $4 \mathrm{Cd}$ to $1 \mathrm{E}(\mathrm{E}=\mathrm{Te}$ or $\mathrm{Se})$ feed molar ratio, and a Cd concentration of $120 \mathrm{mmol} \cdot \mathrm{kg}^{-1}(96 \mathrm{mM})$ in OLA. The Cd precursor solution at $120^{\circ} \mathrm{C}$ was mixed with the $\mathrm{E}$ precursor solution, resulting in a total weight of about $5.00 \mathrm{~g}$. The resulting two mixtures were degassed under vacuum until no bubbles were apparent; under a $\mathrm{N}_{2}$ atmosphere, the mixtures were heated for $30 \mathrm{~min}$ at a desired temperature $\left(120-140^{\circ} \mathrm{C}\right)$. Clear solutions were obtained. Occasionally, the samples were stored at a liquid $\mathrm{N}_{2}$ temperature for future use. The CdTe and CdSe samples were mixed at room temperature with a 1:1 volume ratio in a vial. For the formation of CdTeSe MSC-399, incubation was required.

Optical absorption measurements. An aliquot of each sample $(30 \mu \mathrm{L})$ was dispersed in toluene $(3.0 \mathrm{~mL})$. The measurements were performed on a Hitachi UH4150 spectrometer and the spectra were usually collected in the range of $300-600 \mathrm{~nm}$ with a $1 \mathrm{~nm}$ interval. Toluene was measured as a background sample. Quartz cuvettes from Hellma Analytics (with the light path of $10 \mathrm{~mm}$ of $3.5 \mathrm{~mL}$ standard QS cells) were used.

Electrospray ionization mass spectrometry (ESI-MS). An Agilent $6210 \mathrm{~A}$ HPLC-TOF/MS in a positive ion mode was used, with acetonitrile as a mobile phase. For the operation of the instrument and the followed-up data analyses, Agilent Mass Hunter software was used. By a side note, the binary samples were placed in a liquid $\mathrm{N}_{2}$ temperate for 3 days. For their mixture, it was prepared at room temperature and incubated for about $30 \mathrm{~min}$. The resulting mixture sample was then stored in a liquid $\mathrm{N}_{2}$ temperate for 3 days. Before measurements, the frozen binary samples and their mixture were de-frozen at room temperature, and $30 \mu \mathrm{L}$ of each was dispersed in toluene $(3.0 \mathrm{ml})$.

${ }^{113} \mathrm{Cd}$ nuclear magnetic resonance (NMR). As-synthesized CdTe and CdSe samples $(0.3 \mathrm{~mL})$, together with their mixture $(0.3 \mathrm{~mL})$, were diluted with $0.3 \mathrm{~mL}$ toluene- $d_{8}$ in a glovebox. The mixture was prepared with the same volumes of the two binary samples at room temperature, and was incubated for $15 \mathrm{~min}$. Each of the spectra was collected with a Bruker Avance III $400 \mathrm{MHz}$, with $\mathrm{Cd}\left(\mathrm{ClO}_{4}\right)_{2}$ as a chemical shift reference and a scanning number of $4096^{24}$. It took about $4 \mathrm{~h}$ to collect each of the spectra. See Supplementary Table 1 for details.

Synchrotron-based in situ small angle X-ray scattering (SAXS). The binary $\mathrm{CdTe}\left(130^{\circ} \mathrm{C} / 30 \mathrm{~min}\right)$ and $\mathrm{CdSe}\left(140^{\circ} \mathrm{C} / 30 \mathrm{~min}\right)$ samples were used (with the $\mathrm{Cd}$ concentration of $96 \mathrm{mM}$ ). They were then stored in a liquid $\mathrm{N}_{2}$ temperature overnight. The next day, the binary samples were mixed at room temperature with equal volumes. The SAXS measurements were carried out at room temperature after the samples added into sample holders with a light pathlength of about $1.0 \mathrm{~mm}$; no dilution was performed. Again ${ }^{24}$, the BL16B1 beamline at Shanghai Synchrotron Radiation Facility (SSRF), Shanghai, China, was used, with X-rays of a wavelength of $\lambda=1.03 \AA$ (energy of $12 \mathrm{keV}$ ) as the incident beam. A Rayonix SX165 CCD detector (Rayonix, Evanston, IL, USA) with a resolution of $2048 \times 2048$ pixels and a pixel size of $80 \mu \mathrm{m} \times 80 \mu \mathrm{m}$ was used to record the scattering intensity. Importantly, all of the data were corrected for background and air scattering. Similarly ${ }^{24}$, the two-dimensional pattern was integrated to obtain the onedimensional SAXS profile with Fit2D software. The detailed information about SAXS data fitting with a model developed by Beaucage can be found elsewhere ${ }^{24}$.

\section{Data availability}

The authors declare that all relevant data supporting the findings of this study are available from the authors on request.

Received: 8 October 2018 Accepted: 22 March 2019

Published online: 11 April 2019

\section{References}

1. Yang, J. et al. Chemical synthesis, doping, and transformation of magic-sized semiconductor alloy nanoclusters. J. Am. Chem. Soc. 139, 6761-6770 (2017).

2. Wang, R. et al. Homogeneously-alloyed CdTeSe single-sized nanocrystals with bandgap photoluminescence. J. Phys. Chem. C 113, 3402-3408 (2009).

3. Murray, C. B., Norris, D. J. \& Bawendi, M. G. Synthesis and characterization of nearly monodisperse $\mathrm{CdE}(\mathrm{E}=\mathrm{S}, \mathrm{Se}, \mathrm{Te})$ semiconductor nanocrystallites. J. Am. Chem. Soc. 115, 8706-8715 (1993).

4. Peng, Z. A. \& Peng, X. Formation of high-quality CdTe, CdSe, and CdS nanocrystals using CdO as precursor. J. Am. Chem. Soc. 123, 183-184 (2001).

5. Yang, Y. A., Wu, H., Williams, K. R. \& Cao, Y. C. Synthesis of CdSe and CdTe nanocrystals without precursor injection. Angew. Chem. Int. Ed. 44, 6712-6715 (2005).

6. Smith, A. M. \& Nie, S. Bright and compact alloyed quantum dots with broadly tunable near-infrared absorption and fluorescence spectra through mercury cation exchange. J. Am. Chem. Soc. 133, 24-26 (2011).

7. Ratcliffe, C. I. et al. Solid state NMR studies of photoluminescent cadmium chalcogenide nanoparticles. Phys. Chem. Chem. Phys. 8, 3510-3519 (2006). 3510.

8. Peng, Z. A. \& Peng, X. Nearly Monodisperse and shape-controlled CdSe nanocrystals via alternative routes: nucleation and growth. J. Am. Chem. Soc. 124, 3343-3353 (2002).

9. Kasuya, A. et al. Ultra-stable nanoparticles of CdSe revealed from mass spectrometry. Nat. Mater. 3, 99-102 (2004).

10. Kudera, S. et al. Sequential growth of magic-size CdSe nanocrystals. $A d v$. Mater. 19, 548-552 (2007).

11. Wang, R. et al. Single-sized colloidal CdTe nanocrystals with strong bandgap photoluminescence. Chem. Commun. 0, 962-964 (2009).

12. Wang, Y., Zhou, Y., Zhang, Y. \& Buhro, W. E. Magic-size II-VI nanoclusters as synthons for flat colloidal nanocrystals. Inorg. Chem. 54, 1165-1177 (2015).

13. Dukes, A. D., McBride, J. R. \& Rosenthal, S. J. Synthesis of magic-sized CdSe and CdTe nanocrystals with diisooctylphosphinic acid. Chem. Mater. 22, 6402-6408 (2010).

14. Hsieh, T. E. et al. Unraveling the structure of magic-size $(\mathrm{CdSe})_{13}$ cluster pairs. Chem. Mater. 30, 5468-5477 (2018)

15. Nevers, D. R. et al. Mesophase formation stabilizes high-purity magic-sized clusters. J. Am. Chem. Soc. 140, 3652-3662 (2018).

16. Zhang, J. et al. Individual pathways in the formation of magic-size clusters and conventional quantum dots. J. Phys. Chem. Lett. 9, 3660-3666 (2018).

17. Owen, J. S., Chan, E. M., Liu, H. \& Alivisatos, A. P. Precursor conversion kinetics and the nucleation of cadmium selenide nanocrystals. J. Am. Chem. Soc. 132, 18206-18213 (2010).

18. LaMer, V. K. \& Dinegar, R. H. Theory, production and formation of monodispersed hydrosols. J. Am. Chem. Soc. 72, 2494-2494 (1950).

19. García-Rodríguez, R., Hendricks, M. P., Cossairt, B. M., Liu, H. \& Owen, J. S. Conversion reactions of cadmium chalcogenide nanocrystal precursors. Chem. Mater. 25, 1233-1249 (2013).

20. Yu, K. et al. General low-temperature reaction pathway from precursors to monomers before nucleation of compound semiconductor nanocrystals. Nat. Commun. 7, 12223 (2016)

21. Wang, L. et al. Precursor self-assembly identified as a general pathway for colloidal semiconductor magic-size clusters. Adv. Sci. 5, 1800623 (2018).

22. Zhu, T. et al. Two-step nucleation of CdS magic-size nanocluster MSC-311. Chem. Mater. 29, 5727-5735 (2017).

23. Luan, C. et al. Evolution of two types of CdTe magic-size clusters from one induction period sample. J. Phys. Chem. Lett. 9, 5288-5295 (2018).

24. Liu, M. et al. Probing intermediates of the induction period prior to nucleation and growth of semiconductor quantum dots. Nat. Commun. 8, 15467 (2017).

25. Zhu, D. et al. Interpreting the ultraviolet absorption in the spectrum of 415 nm-bandgap CdSe magic-size clusters. J. Phys. Chem. Lett. 9, 2818-2824 (2018).

26. Zhang, B. et al. Thermally-induced reversible structural isomerization in colloidal semiconductor CdS magic-size clusters. Nat. Commun. 9, 2499 (2018).

27. Saruyama, M. et al. Spontaneous formation of wurzite-CdS/zinc blende-CdTe heterodimers through a partial anion exchange reaction. J. Am. Chem. Soc. 133, 17598-17601 (2011).

28. Li, J., Jia, J., Lin, Y. \& Zhou, X. Controlled optical properties of water-soluble CdTe nanocrystals via anion exchange. J. Colloid. Interface. Sci. 463, 69-74 (2016).

29. Sines, I. T. et al. Engineering porosity into single-crystal colloidal nanosheets using epitaxial nucleation and chalcogenide anion exchange reactions: the conversion of SnSe to SnTe. Chem. Mater. 24, 3088-3093 (2012).

30. Park, J., Zheng, H., Jun, Y. W. \& Alivisatos, A. P. Hetero-epitaxial anion exchange yields single-crystalline hollow nanoparticles. J. Am. Chem. Soc. 131 13943-13945 (2009).

31. Dloczik, L. \& Könenkamp, R. Nanostructure transfer in semiconductors by ion exchange. Nano. Lett. 3, 651-653 (2003). 
32. Son, D. H., Hughes, S. M., Yin, Y. \& Alivisatos, A. P. Cation exchange reactions in ionic nanocrystals. Science 306, 1009-1012 (2004).

33. Zhang, J., Tang, Y., Lee, K. \& Ouyang, M. Nonepitaxial growth of hybrid coreshell nanostructures with large lattice mismatches. Science 327, 1634-1638 (2010).

34. De Trizio, L. \& Manna, L. Forging colloidal nanostructures via cation exchange reactions. Chem. Rev. 116, 10852-10887 (2016).

35. Fenton, J. L., Steimle, B. C. \& Schaak, R. E. Exploiting crystallographic regioselectivity to engineer asymmetric three-component colloidal nanoparticle isomers using partial cation exchange reactions. J. Am. Chem. Soc. 140, 6771-6775 (2018).

36. Fenton, J. L., Steimle, B. C. \& Schaak, R. E. Tunable intraparticle frameworks for creating complex heterostructured nanoparticle libraries. Science $\mathbf{3 6 0}$, 513-517 (2018).

37. Capps, K. B., Wixmerten, B., Bauer, A. \& Hoff, C. D. Thermochemistry of sulfur atom transfer. Enthalpies of reaction of phosphines with sulfur, selenium, and tellurium, and of desulfurization of triphenylarsenic sulfide, triphenylantimony sulfide, and benzyl trisulfide. Inorg. Chem. 37, 2861-2864 (1998).

38. Cox, G. D., Wagman, D. D. \& Mwdvedev, V. A. CODATA Key values for thermodynamics. (Hemisphere Publishing, New York, 1989).

39. De Hoffmann, E. \& Stroobant, V. Mass spectrometry. principles and applications 3rd edn. (Wiley, West Sussex, 2007).

40. Krishnadas, K. R., Baksi, A., Ghosh, A., Natarajan, G. \& Pradeep, T. Structureconserving spontaneous transformations between nanoparticles. Nat. Commun. 7, 13447 (2016).

41. Wang, Y. et al. Isolation of the magic-size CdSe nanoclusters $\left[(\mathrm{CdSe})_{13}(\mathrm{n}-\right.$ octylamine $\left.)_{13}\right]$ and $\left.\left[(\mathrm{CdSe})_{13} \text { (oleylamine }\right)_{13}\right]$. Angew. Chem. Int. Ed. 51, 6154-6157 (2012).

42. Muckel, F. et al. Digital doping in magic-sized CdSe clusters. ACS Nano 10, 7135-7141 (2016).

43. Wurmbrand, D., Fischer, J. W. A., Rosenberg, R. \& Boldt, K. Morphogenesis of anisotropic nanoparticles: self-templating via non-classical, fibrillar $\mathrm{Cd} 2 \mathrm{Se}$ intermediates. Chem. Commun. 54, 7358-7361 (2018).

44. Li, T., Senesi, A. J. \& Lee, B. Small angle X-ray scattering for nanoparticle research. Chem. Rev. 116, 11128-11180 (2016).

45. Beaucage, G., Kammler, H. K. \& Pratsinis, S. E. Particle size distributions from small-angle scattering using global scattering functions. J. Appl. Crystallogr. 37, 523-535 (2004).

46. Pickard, C. J. \& Needs, R. J. Ab initio random structure searching. J. Phys. Condens. Matter. 23, 053201 (2011).

47. Liu, Y. et al. Effect of small molecule additives in the prenucleation stage of semiconductor CdSe quantum dots. J. Phys. Chem. Lett. 9, 6356-6363 (2018).

48. Kirkwood, N. \& Boldt, K. Protic additives determine the pathway of CdSe nanocrystal growth. Nanoscale 10, 18238-18248 (2018).

49. Landes, C., Braun, M., Burda, C. \& El-Sayed, M. A. Observation of large changes in the band gap absorption energy of small CdSe nanoparticles induced by the adsorption of a strong hole acceptor. Nano. Lett. 1, 667-670 (2001).

50. Steckel, J. S., Yen, B. K., Oertel, D. C. \& Bawendi, M. G. On the mechanism of lead chalcogenide nanocrystal formation. J. Am. Chem. Soc. 128, 13032-13033 (2006).

51. Evans, C. M., Evans, M. E. \& Krauss, T. D. Mysteries of TOPSe revealed: insights into quantum dot nucleation. J. Am. Chem. Soc. 132, 10973-10975 (2010).

52. $\mathrm{Yu}, \mathrm{K}$. et al. The formation mechanism of binary semiconductor nanomaterials: shared by single-source and dual-source precursor approaches. Angew. Chem. Int. Ed. 52, 4823-4828 (2013).
53. Garcia-Rodriguez, R. \& Liu, H. Solution structure of cadmium carboxylate and its implications for the synthesis of cadmium chalcogenide nanocrystals. Chem. Commun. 49, 7857-7859 (2013).

54. Garcia-Rodriguez, R. \& Liu, H. A nuclear magnetic resonance study of the binding of trimethylphosphine selenide to cadmium oleate. J. Phys. Chem. A 118, 7314-7319 (2014).

55. Wang, F., Richards, V. N., Shields, S. P. \& Buhro, W. E. Kinetics and mechanisms of aggregative nanocrystal growth. Chem. Mater. 26, 5-21 (2014).

\section{Acknowledgements}

K.Y. thanks National Natural Science Foundation of China (NSFC) 21573155 and 21773162, the Fundamental Research Funds for the Central Universities SCU2015A002, the State Key Laboratory of Polymer Materials Engineering of Sichuan University (Grant No. sklpme2018-2-08), and the Open Project of Key State Laboratory for Supramolecular Structures and Materials of Jilin University for SKLSSM 201830. J.Z. is grateful to National Natural Science Foundation of China (NSFC) 11305242. We thank Miss Juan Zhang for her exploration on the experimental conditions for the alloy synthesis in the initial stage of the project. We thank Miss Yuanyuan Liu for TEM and Sichuan Univ of Analytical \& Testing Center for NMR.

\section{Author contributions}

K.Y. designed and directed this study. D.G. and H.Z. carried out the synthesis, M.Z. assisted MS, C.Z. did NMR, X.H. and J.Z. performed SAXS, while D.L. and Y.J. supported XPS. All authors contributed to data analysis and writing and editing of the manuscript.

\section{Additional information}

Supplementary Information accompanies this paper at https://doi.org/10.1038/s41467019-09705-w.

Competing interests: The authors declare no competing interests.

Reprints and permission information is available online at http://npg.nature.com/ reprintsandpermissions/

Journal peer review information: Nature Communications would like to thank Haitao Liu, Yuanyuan Wang, and the other anonymous reviewer(s) for their contribution to the peer review of this work.

Publisher's note: Springer Nature remains neutral with regard to jurisdictional claims in published maps and institutional affiliations.

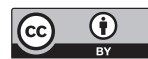

Open Access This article is licensed under a Creative Commons Attribution 4.0 International License, which permits use, sharing, adaptation, distribution and reproduction in any medium or format, as long as you give appropriate credit to the original author(s) and the source, provide a link to the Creative Commons license, and indicate if changes were made. The images or other third party material in this article are included in the article's Creative Commons license, unless indicated otherwise in a credit line to the material. If material is not included in the article's Creative Commons license and your intended use is not permitted by statutory regulation or exceeds the permitted use, you will need to obtain permission directly from the copyright holder. To view a copy of this license, visit http://creativecommons.org/ licenses/by/4.0/.

(C) The Author(s) 2019 